\title{
Shot noise in the chaotic-to-regular crossover regime
}

\author{
Florian Aigner, Stefan Rotter, and Joachim Burgdörfer \\ Institute for Theoretical Physics, Vienna University of Technology, A-1040 Vienna, Austria
}

(Dated: November 17, 2018)

\begin{abstract}
We investigate the shot noise for phase-coherent quantum transport in the chaotic-to-regular crossover regime. Employing the Modular Recursive Green's Function Method for both ballistic and disordered two-dimensional cavities we find the Fano factor and the transmission eigenvalue distribution for regular systems to be surprisingly similar to those for chaotic systems. We argue that in the case of regular dynamics in the cavity, diffraction at the lead openings is the dominant source of shot noise. We also explore the onset of the crossover from quantum to classical transport and develop a quasi-classical transport model for shot noise suppression which agrees with the numerical quantum data.
\end{abstract}

PACS numbers: 73.23.-b, 05.45.Mt, 73.63.Kv, 72.70.+m

The significance of noise induced by the discreteness of the electron charge ("shot noise") first investigated almost a century ago 1] has resurfaced in the field of mesoscopic physics 2, 3]. Shot noise carries information about the crossover from a deterministic (classical) particle picture of electron motion to a probabilistic (quantum) description, where electrons behave as matter waves. The uncertainty inherent in a quantum picture gives rise to noisy transport. In conductance through quantum dots the correlations between electrons in the Fermi sea lead to a suppression of shot noise $S$ relative to the Poissonian value of uncorrelated electrons $S_{P}$ [4]. The reduction relative to the completely random limit is customarily expressed in terms of the Fano factor $F=S / S_{P}$.

Most investigations to date have focused on quantum dots whose classical dynamics is fully chaotic 4, 5, 6. 7, 8, 9, 10, 11]. In this limit, random matrix theory (RMT) 8] predicts a universal value for the Fano factor, $F=1 / 4$. The applicability of this RMT result requires, in addition to the underlying chaotic dynamics, dwell times in the open cavity $\tau_{D}$ which are sufficiently long compared to the Ehrenfest time $\tau_{E}$. The latter estimates the time for the initially localized quantum wavepackets to spread all over the width $d$ of the cavity (typically $d \approx \sqrt{A}$ with $A$ area of the dot) due to the divergence of classical chaotic trajectories. It can be estimated as 12

$$
\tau_{E}=\Lambda^{-1} \ln \left(d / \lambda_{F}\right),
$$

where $\Lambda$ is the Lyapunov exponent $(\Lambda>0$ for a chaotic cavity), and $\lambda_{F}$ is the de Broglie wavelength associated with the wavenumber at the Fermi surface $k_{F}$. The limit $\tau_{E} / \tau_{D} \ll 1$ corresponds to the quantum (or RMT) regime and $\tau_{E} / \tau_{D} \gg 1$ corresponds to the classical limit for which $F=0$ is expected. For ballistic cavities in the crossover between these two regimes a simple conjecture for $F$ was put forward [7],

$$
F=1 / 4 \exp \left(-\tau_{E} / \tau_{D}\right)
$$

For cavities with a short-ranged disorder potential, an alternative crossover behavior,

$$
F=1 / 4\left(1+\tau_{Q} / \tau_{D}\right)^{-1},
$$

was proposed [13, 14], where $\tau_{Q}$ is a characteristic scattering time within which the wavepacket is scattered into random direction and thus explores the entire cavity. $\tau_{Q}$ and $\tau_{E}$ are closely related to another as both denote the characteristic time scale for spreading of the wavepacket by chaotic scattering either at the boundary $\left(\tau_{E}\right)$ or the interior $\left(\tau_{Q}\right)$ of the cavity. Moreover, for short ranged disorder with a correlation length $l_{C}<\lambda_{F}, \tau_{Q}$ incorporates, just as $\tau_{E}$, quantum effects and depends on an effective $\hbar_{\text {eff }}$ of the system. The crossover from the chaotic to the regular regime is therefore predicted to be controlled by a single ratio $\tau_{E} / \tau_{D}$ or $\tau_{Q} / \tau_{D}$ which will be a function of both the size of quantum effects $\left(\hbar_{\text {eff }}\right)$ and the mean rate of irregular (chaotic) scattering, $\langle\Lambda\rangle$. The chaoticto-regular crossover corresponds to the limit $\langle\Lambda\rangle \rightarrow 0$, while the quantum-to-classical limit involves $\hbar_{\mathrm{eff}} \rightarrow 0$.

An obvious test of different predictions would be to simulate phase-coherent scattering processes on a computer. However, conventional numerical techniques suffer from a slow convergence rate for large $\tau_{E}$ and, in particular, for small $\hbar_{\mathrm{eff}}$. To circumvent this difficulty an "open" dynamical kicked rotator model was recently successfully used to mimic chaotic scattering in a 1D-system [6, 10]. Experimental tests have, so far, been limited to the regime of $\tau_{Q} / \tau_{D}<1$ [14].

One open question not yet well understood is the behavior of shot noise for motion in a regular rather than chaotic cavity, i.e. in the limit $\Lambda \rightarrow 0$. For a mixed system lower values of $F$ have been observed [15] suggesting that for regular systems $F$ may vanish. Taken at face value, Eq. (2) yields $F=0$ for the case of $\tau_{E} \rightarrow \infty$ or $\Lambda \rightarrow 0$ at fixed value of $\hbar_{\mathrm{eff}}$. This result would correspond to a complete suppression of shot noise. On the other hand, the notion of noisy transport as a result of the wave nature of electrons would suggest that noise should be inherent in phase-coherent transport through mesoscopic structures irrespective of the chaoticity of the underlying clas- 


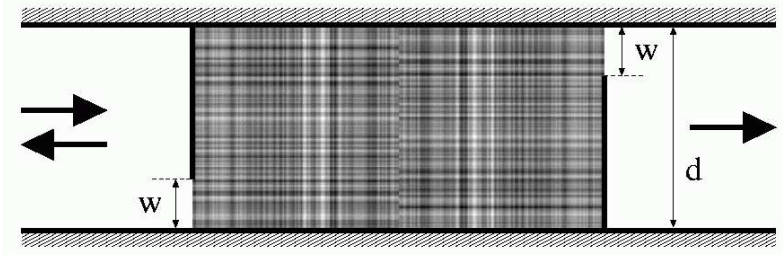

FIG. 1: Rectangular quantum billiard with tunable shutters and tunable disorder potential (gray shaded area). Electrons are injected from the left into the cavity region of size $A=2 d^{2}$, width $2 d$ and height $d$. Tuning the opening of the shutters $w$ and the strength of the disorder potential $V_{0}$, the onset of the crossover from quantum-to-classical and chaoticto-regular scattering can be investigated, respectively.

sical dynamics. In the present letter we analyze a model for transport through a cavity that allows to investigate the crossover regime from chaotic to regular dynamics, i.e. $\Lambda \rightarrow 0$, and the onset of the crossover from quantum to classical dynamics. In our model, the dwell time and the mean rate of chaotic spreading of the wavepacket, $\langle\Lambda\rangle$, can be tuned independently. Surprisingly, we find that the Fano factor and the distribution of transmission eigenvalues for regular systems resemble closely that of chaotic systems. We interpret this observation in terms of the ubiquity and dominance of diffractive scattering in phase-coherent cavities.

We choose first a scattering geometry (Fig. 1) which consists of a rectangular cavity to which two leads of width $d$ are attached via tunable shutters with an opening width $w$. To reduce direct transport between the shutter openings, they are placed on the bottom and top end of the leads, respectively. The cavity region of width $d$ and length $2 d$ contains a disorder potential $V$ characterized by its mean value $\langle V\rangle=0$, and the correlation function $\langle V(x) V(x+a)\rangle=\left\langle V^{2}\right\rangle \exp \left(-a / l_{C}\right)$ [16]. The correlation length $l_{C}$ is typically a small fraction of the Fermi wavelength $l_{C} / \lambda_{F} \approx 0.12$ and the potential strength $V_{0}=\sqrt{\left\langle V^{2}\right\rangle}$ is weak, $V_{0} / E_{F} \leq 0.1$. The dependence of $\tau_{D}$ on the shutter opening was determined by a Monte-Carlo sampling of $\sim 10^{5}$ classical trajectories, $\tau_{D} \approx 2.66 /\left(w k_{F}\right)$, i.e. approximately independent of $V_{0}$. In the limit of vanishing disorder potential $\left(V_{0} \rightarrow 0\right)$ the motion inside the cavity becomes completely regular.

Our quantum calculation proceeds within the framework of the modular recursive Green's function method (MRGM) 17] which allows to treat two-dimensional quantum dots with relatively small $\lambda_{F}$ (or small $\hbar_{\text {eff }}$ ). The MRGM requires, however, separable modules. We construct the latter by decomposing the cavity region into two square modules for each of which we choose a separable random potential $V(x, y)=V_{1}(x)+V_{2}(y)$. In order to destroy the unwanted separability and to ensure chaotic dynamics, we build up the cavity by combining two identical modules, however, rotated by $180^{\circ}$ relative to each other (see Fig. 1 for illustration). As the Fermi wavenumber and the width of the leads $d$ is independent of the shutter openings $w$, this device allows to study two different crossovers of shot noise: (1) By changing the opening of the shutters $w$ the dwell time in the cavity $\tau_{D}$ can be tuned without changing the Fermi energy $E_{F}$. With the increase of $w$ the onset of the quantumto-classical crossover can be probed, with the sideffect, however, that not only $\hbar_{\text {eff }}$ is reduced but simultaneously the classical phase space structure is altered. This closely resembles the parameter tuning in the experiment 14]. (2) By tuning the strength of the disorder potential, the rate of chaotic spreading, $\langle\Lambda\rangle$, and thus $\tau_{E}$ (or $\tau_{Q}$, used in the following interchangeably) is varied.

We evaluate the transmission amplitudes $t_{m n}$ for an electron injected from the left by projecting the Green's function at the Fermi energy $G\left(E_{F}\right)$ onto all modes $m, n \in[1, \ldots, N]$ in the in- and outgoing lead, respectively. The Fano factor $F$ is then calculated from the $N$-dimensional transmission matrices $t[\underline{3}]$,

$$
F=\frac{\left\langle\operatorname{Tr} \mathrm{t}^{\dagger} \mathrm{t}\left(\mathbb{1}-\mathrm{t}^{\dagger} \mathrm{t}\right)\right\rangle}{\left\langle\operatorname{Tr} \mathrm{t}^{\dagger} \mathrm{t}\right\rangle}=\frac{\left\langle\sum_{n=1}^{N} T_{n}\left(1-T_{n}\right)\right\rangle}{\left\langle\sum_{n=1}^{N} T_{n}\right\rangle},
$$

with $T_{n}$ being the eigenvalues of $t^{\dagger} t$. The brackets $\langle\ldots\rangle$ indicate that we average over 150 equidistant points in the wavenumber-range $k_{F} \in[40.1,40.85] \times \pi / d$, where 40 transverse lead modes are open. Note that this mode number is higher than in previous studies. We choose a nearest-neighbor spacing $\Delta x=\Delta y$ in the Cartesian discretization grid such that the Fermi wavelength is well resolved by a large number of grid points, $\lambda_{F} \approx 32 \Delta x$. With these settings we have a total number of $\sim 8.5 \times 10^{5}$ grid points in the interior of the cavity.

Figure 2 displays the Fano factor as a function of the inverse dwell time $\tau_{D}^{-1}$ (Fig. 2a) and the inverse Ehrenfest time $\tau_{E}^{-1}$ (Fig. 2b). For $\tau_{D}^{-1} \rightarrow 0$ (i.e. large dwell times) $F$ approaches the universal value $1 / 4$ irrespective of the strength of the disorder potential $V_{0}$, while for shorter dwell times $F$ falls off gradually (Fig. 2 a). The steepness of this decrease is clearly dependent on $V_{0}$ and thus on the mean scattering rate $\langle\Lambda\rangle$. Most striking is the feature that for $V_{0} \rightarrow 0$ but long dwell times the shot noise reaches the RMT value even though the dynamics is now entirely regular (see Fig. 2a). In Fig. 20 this feature is reflected by the fact that the Fano factor $F$ does not decay to zero even as $V_{0} \rightarrow 0$ (i.e. $\tau_{E}^{-1} \rightarrow 0$ ). There is no compelling a priori reason why the RMT prediction should be applicable to regular systems. This observation suggests that the conjectures [Eq. (2) or Eq. (3)] require a modification to properly account for the shot noise in the regular limit. We argue that the key point is the wavepacket diffraction at the cavity openings which has to be incorporated in the theoretical description of shot noise. Note that this feature is inherent in quantum 

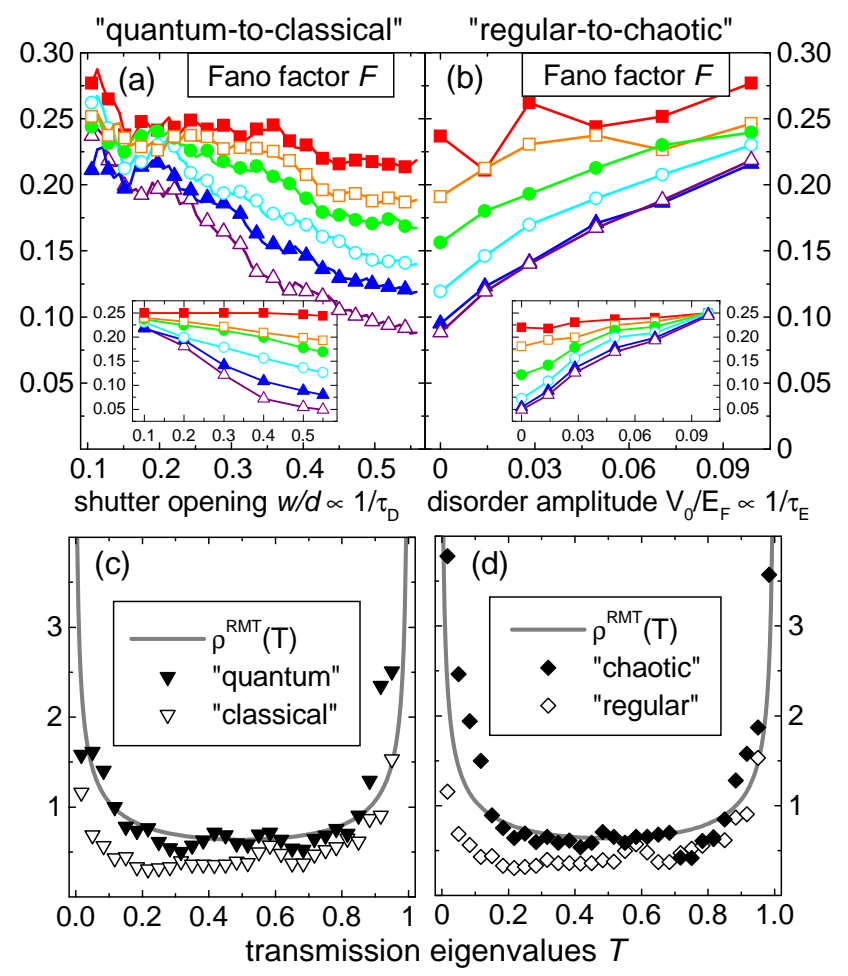

FIG. 2: (a) Fano factor $F$ in the quantum-toclassical crossover regime for the cavity depicted in Fig. 11 Curves shown correspond to different strengths of the disorder potential: $V_{0} / E_{F}=$

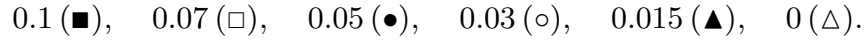
(b) Regular-to-chaotic crossover regime of $F$. Curves corresponding to different shutter opening ratios are shown:

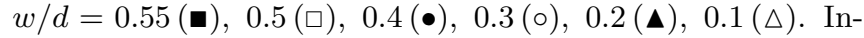
sets of (a) and (b) depict the fit parameter free prediction with diffractive corrections [Eq. (5] ]. (c) Distribution of transmission eigenvalues $\rho(T)$ in the quantum-to-classical crossover: $V_{0} / E_{F}=0(\mathbf{\nabla}), 0(\nabla)$ and $w / d=15(\mathbf{\nabla}), 50(\nabla)$. (d) $\rho(T)$ in the chaotic-to-regular crossover: $V_{0} / E_{F}=0.1(\diamond), 0(\diamond)$ and $w / d=50(\diamond), 50(\diamond)$.

transport and independent of the underlying regular or chaotic dynamics 17]. Scattering due to chaotic dynamics, which lies at the core of RMT, certainly leads to wavepacket spreading but does not constitute the only or, in general, dominant source.

Diffraction at the cavity openings has been studied in detail [18, 19] and can be described by a standard Fraunhofer diffraction analysis for electrons which enter or leave the cavity [18]. An estimate for wavepacket spreading can be found by considering the characteristic angular injection patterns of the transverse modes in these openings. Averaging the individual patterns over the total number of modes in the shutter openings, $M=$ $\operatorname{int}\left(k_{F} w / \pi\right)$, we obtain for the variance of the injection angle $\theta$ the dependence: $\left\langle\sqrt{\left\langle\theta^{2}\right\rangle-\langle\theta\rangle^{2}}\right\rangle \approx 0.5 \times M^{-0.5}$. This result now enters our considerations on a modified estimate for $\tau_{E}\left(\right.$ or $\left.\tau_{Q}\right)$. We perform a quasi-classical Monte-Carlo transport simulation [20] in which we follow an ensemble of classical trajectories subject to a random Poissonian scattering process. For the latter we calculate the transport mean free path $\left(\tau_{S} \cdot v_{F}\right)$ and the differential scattering probability $(P(\theta) \sim d \sigma / d \theta)$ in first Born approximation, thus taking into account quantum diffractive scattering (for $l_{C} \cdot k_{F}<1$ ) along the lines of Refs. 13, 14]. In this simulation, spreading results from both the injection of classical trajectories with an initial angular distribution given by Fraunhofer diffraction at the shutter opening and multiple scattering inside the cavity. This allows to identify a modified Ehrenfest time $\widetilde{\tau}_{E}$ as the time it takes for the ensemble to acquire a mean spread of the order of the cavity width $d$ under the influence of both disorder (or chaotic) scattering inside the cavity as well as diffraction at the shutter openings. At $\widetilde{\tau}_{E}$ the mean separation reaches $\Delta r\left(\widetilde{\tau}_{E}\right)=\left\langle(\vec{r}-\langle\vec{r}\rangle)^{2}\right\rangle^{1 / 2}=d$. Already the inclusion of Fraunhofer scattering alone leads to a drastic reduction of the Ehrenfest time: Injected electrons spread much faster over the whole cavity than according to the estimate in Eq. (11). An important consequence of this modification is that the parameter $\widetilde{\tau}_{E}$ is now of comparable magnitude or even smaller than the time $\tau_{0}$ beyond which universal behavior for $P(t)$ sets in $\left[P(t) \propto \exp \left(-t / \tau_{D}\right)\right.$ for chaotic and $P(t) \propto\left(t / \tau_{D}\right)^{-\beta}$ for regular dots]. In this regime system-specific deviations of the dwell time distribution from a universal decay law are more pronounced than characteristic mean differences between regular and chaotic cavities 17, 18, 19]. For our estimate of the Fano factor $F$ we therefore take into account the exact dwell time distribution $P(t)$, resulting from the quasi-classical Monte-Carlo trajectory simulation for the particular system we study. Following the analysis [9] based on the contribution of noiseless transmission channels, we find

$$
F=1 / 4\left[1-\int_{0}^{\tilde{\tau}_{E}} P(t) d t\right]=1 / 4 \int_{\widetilde{\tau}_{E}}^{\infty} P(t) d t .
$$

Note that Eq. (5) is applicable to chaotic and regular systems and is expected to be valid irrespective of whether the origin of spreading is ballistic scattering at the boundary or diffractive scattering inside of the cavity.

It is now tempting to probe the convergence towards the RMT limit for $\widetilde{\tau}_{E} / \tau_{D}<1$ also for the distribution of transmission eigenvalues $\rho(T)$ and for other regular and chaotic structures that feature only ballistic motion. We therefore investigate transport across the semi-circular, rectangular and semi-stadium shaped cavity which are prototypical for regular and chaotic ballistic motion, respectively (see insets of Fig. 3 3b,c,d). Unlike in the case of the geometry of Fig. [1] where the number of open modes was fixed, transport coefficients were calculated here for a wide range of $k_{F}$. The dynamics of these cavities is classically scaling invariant. An increase of $k_{F}$ thus probes 

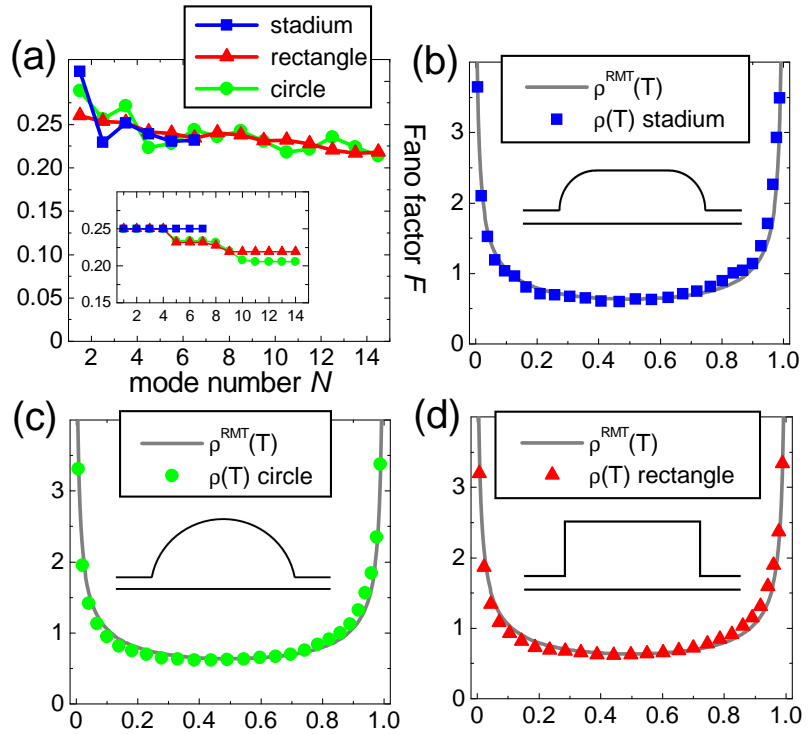

FIG. 3: (a) Fano factor $F$ for scattering systems with chaotic (stadium) and regular (circle, rectangle) classical dynamics as a function of $k_{F}$, in units of the number of open modes $N=\operatorname{int}\left(k_{F} b / \pi\right)$ (each data point is averaged over 200 equidistant $k_{F}$-values). The inset shows the fit parameter free prediction with diffractive corrections [see Eq. (5)]. (b)-(d) Distribution of transmission eigenvalues $\rho(T)$ averaged over the $k_{F}$-range depicted in (a). Remarkably the obtained values for $\rho(T)$ correspond very closely to the RMT-prediction $\rho^{\mathrm{RMT}}(T)$, irrespective of the chaoticity in the cavity. The insets show the cavity geometries.

directly the dependence of $\widetilde{\tau}_{E} / \tau_{D}$ on $\hbar_{\text {eff }}$, while keeping the classical phase space and $\Lambda$ fixed. Unlike the $\tau_{D} \rightarrow 0$ crossover performed with the help of the tunable shutters as in the experiment [14] and in the simulation above, the $k_{F} \rightarrow \infty$ limit would correspond to the "pure" quantumto-classical crossover as $\hbar_{\mathrm{eff}} \rightarrow 0$.

Employing the same high density of grid points we reach up to 15 flux-carrying modes in the semi-circular and rectangular billiard and 7 open lead modes in the case of the semi-stadium [17]. We therefore can observe only the onset of the quantum-to-classical crossover for these three cavities. Their area $A=(4+\pi) / 2$ and lead width $b=0.125$ are identical. Given this small leadwidth, i.e. $b / \sqrt{A}=0.066$, our results agree very well with those shown in Fig. 2 for small shutter openings: at all energies the Fano factor is close to $1 / 4$, irrespective of the chaoticity in the cavity. However, for a higher number of open modes (i.e. higher $k_{F}$ ) diffraction becomes less important since $\widetilde{\tau}_{E}$ becomes larger and a decrease in the Fano factor ensues, signifying the onset of the quantumto-classical crossover. Our numerical data (Fig. 3 a) agree indeed with this prediction. Moreover, the quantitative behavior is well described by Eq. (5) (see inset of Fig. 3h). The RMT prediction for the distribution of transmission eigenvalues $T_{n}$ is given by [8] $\rho^{\mathrm{RMT}}(T)=$
$1 /[\pi \sqrt{T(1-T)}]$. Taking our original scattering geometry (Fig. 1) with a setting of wide shutter openings (short $\tau_{D}$ or "classical") and no disorder potential ("regular") we find a curve which clearly deviates from this RMTprediction (see Fig. 2r,d). Remarkably, the RMT-limit is, however, restored equally well by either (1) further closing the shutters (longer $\tau_{D}$ or "quantum") or (2) by increasing the disorder potential (increase of $\langle\Lambda\rangle$ or "chaotic"). In line with this observation the stadiumshaped, circular and rectangular cavity all fulfill the $\rho^{\mathrm{RMT}}(T)$ remarkably well (see Fig. Bb,c,d) due to their small lead openings (i.e. long dwell times). This suggests that for $\widetilde{\tau}_{E} / \tau_{D} \ll 1$ not just $F$ but also the distribution function $\rho(T)$ approaches the "universal" RMT limit.

To summarize, we have numerically verified the behavior of the Fano factor $F$ in a realistic scattering system with a tunable disorder potential and tunable shutters. We find that diffractive quantum scattering is sufficient to establish the RMT eigenvalue distribution $\rho^{\mathrm{RMT}}(T)$, irrespective of regular or chaotic dynamics. The chaoticto-regular and quantum-to-classical crossover in $F$ can be estimated by a generalization of a previously proposed dependence [9] on the Ehrenfest time $\widetilde{\tau}_{E}$ [Eq. [5]], provided that the definition of the Ehrenfest time is properly modified to include diffraction.

We thank C.W.J. Beenakker, J. Cserti, V.A. Gopar, F. Libisch, I. Rotter, M. Seliger, H. Schomerus, E.V. Sukhorukov and L. Wirtz for helpful discussions. Support by the Austrian Science Foundation (Grant No. FWF-SFB016 and No. FWF-P17359) is gratefully acknowledged.

[1] W. Schottky, Ann. Phys. (Leipzig) 57, 541 (1918).

[2] C.W.J. Beenakker and C. Schönenberger, Physics Today 56(5), 37 (2003).

[3] Ya. M. Blanter and M. Büttiker, Phys. Rep. 336, 1 (2000).

[4] C.W.J. Beenakker and H. van Houten, Phys. Rev. B 43, 12066 (1992).

[5] R.G. Nazmitdinov, H.-S. Sim, H. Schomerus, and I. Rotter, Phys. Rev. B 66, 241302 (2002).

[6] J. Tworzydło, A. Tajic, H. Schomerus, and C. W. J. Beenakker, Phys. Rev. B 68, 115313 (2003).

[7] O. Agam, I. Aleiner, and A. Larkin, Phys. Rev. Lett. 85, 3153 (2000).

[8] H.U. Baranger and P.A. Mello, Phys. Rev. Lett. 73, 142 (1994); R.A. Jalabert, J.-L. Pichard, and C.W.J. Beenakker, Europhys. Lett. 27, 255 (1994).

[9] P.G. Silvestrov, M.C. Goorden, and C.W.J. Beenakker, Phys. Rev. B. 67, 241301(R) (2003).

[10] Ph. Jacquod and E.V. Sukhorukov, Phys. Rev. Lett. 92, 116801 (2004).

[11] E.V. Sukhorukov and O.M. Bulashenko, cond-mat/0408075 (2004).

[12] G.M. Zaslavsky, Phys. Rep. 80, 157 (1981).

[13] S. Oberholzer, Ph.D. thesis (University Basel, 2001). 
[14] S. Oberholzer, E.V. Sukhorukov, and C. Schönenberger, Nature 415, 765 (2002).

[15] H.-S. Sim and H. Schomerus, Phys. Rev. Lett. 89, 66801 (2002).

[16] Th. Koschny, H. Potempa, and L. Schweitzer, Phys. Rev. Lett. 86, 3863 (2001).

[17] S. Rotter et al., Phys. Rev. B 62, 1950 (2000); Phys. Rev. B 68, 165302 (2003).
[18] L. Wirtz, J.-Z. Tang, and J. Burgdörfer, Phys. Rev. B 56, 7589 (1997).

[19] L. Wirtz, C. Stampfer, S. Rotter, and J. Burgdörfer, Phys. Rev. E 67, 016206 (2003).

[20] J. Burgdörfer and J. Gibbons, Phys. Rev. A, 42, 1206 (1990). 